\title{
Long term relation between solar activity and surface temperature at different geographical regions
}

\author{
M. P. Souza-Echer ${ }^{1}$, W. D. Gonzalez ${ }^{1}$, E. Echer ${ }^{1}$, \\ D. J. R. Nordemann ${ }^{1}$ and N. R. Rigozo ${ }^{2}$ \\ ${ }^{1}$ National Institute for Space Research (INPE) \\ S. J. Campos, SP, Brazil \\ email: mariza@dge.inpe.br \\ ${ }^{2}$ CRS, Santa Maria, RS, Brazil \\ email: rigozo@lacesm.ufsm.br
}

\begin{abstract}
Global suface temperature has showed a rise trend in the last 150 years. This has been mainly attributed to the anthropogenic induced grenhouse gases emissions. However, the role of natural processes is not completely understood and should not be underestimated. In this work, we compare the long term variability of solar activity (as quantified by the sunspot number) with several surface temperature series from different geographical regions (global, hemispheric and latitudinal ranges). The interval of analysis is 1880-2005. The data are analyzed with wavelet multiresolution technique. It has been found that the solar activity long term trend has a maximum around 1970, while air surface temperature series showed maximum (still rising) at 2005. There are differences in the long term trend for Northern and Southern hemispheres. These differences and the relation with solar activity are discussed in this work.
\end{abstract}

Keywords. Solar Activity, Climate Change, Sun Climate relation.

\section{Introduction}

The air surface temperature is a basic meteorological parameter and its variation is a primary measure of global, regional and local climate changes. During the last 150 years, an upward trend of $0.6^{\circ} \mathrm{C}$ in the global surface temperature data has been observed, which has been considered to be the main signature of the so called global climatic warming. The largest part of this climatic warming is usually attributed to the anthropogenic effects due to the enhanced grenhouse gases concentrations. However, there seems to be evidence that natural phenomena can account for part of the climate variability (Souza Echer et al. 2009; Souza Echer et al. 2012; Scafetta 2010).

Scafetta (2010) found empirical evidences that the climatical oscillations within the secular scales are likely driven by astronomical cycles. It was also found in that work that in all major surface temperature records there are several cycles that are coincident with astronomical cycles.

The sunspot number variability and the associated solar activity cycle are known to have important impacts in the geomagnetic activity and space weather variability (Echer et al. 2005). Both the solar irradiance variation and geomagnetic disturbances could have some impact on Earth's climate, although this is a topic of intense debate and research (Haigh 2007; Souza Echer et al. 2009). Furthermore, the influence of these natural solar oscillations on the air surface temperature can be dependent of local conditions (Souza Echer et al. 2008; Souza Echer et al. 2012). 

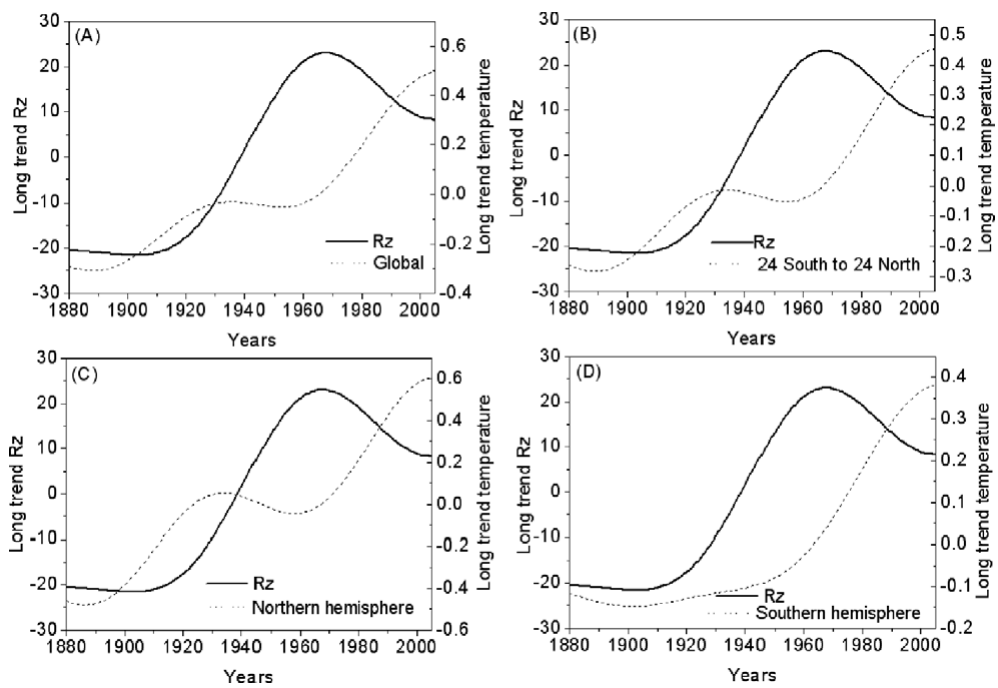

Figure 1. Long term trend for sunspot number (solid line) and for surface temperature (dotted lines). Panels show temperature long term trends for (A),Global, (B) $24^{\circ} \mathrm{N}-24^{\circ} \mathrm{S}$, (C) Northern hemisphere and (D) Southern hemisphere averaged temperatures

In this work, we compare the long term variability of the solar activity, quantified by the sunspot number, with several surface temperature series from different geographical regions (global, hemispheric and latitudinal ranges). The period of study is the interval covering NASA/GISS temperature database, 1880-2005. The data are analyzed with wavelet multi-resolution analysis (MRA).

\section{Methodology and Data}

For studying the terrestrial climate, we have used the compiled NASA/GISS database for global, hemispheric and latitudinal air surface temperature series, available for 18802005 (Hansen \& Lebedeff 1988, Hansen et al. 1996; Hansen et al. 1999).

The longest solar activity index is the sunspot number $\left(\mathrm{R}_{z}\right)$, which was first compiled by R. Wolf in the XIX century and it is available as annual averages since 1700 (Eddy 1976; Hoyt \& Schatten 1997; Echer et al. 2005; Hathaway 2010). The annual averages of $\mathrm{R}_{z}$ used in this work were obtained from the Sunspot Index Data Center and the period used is the same as for the surface temperature series, 1880 to 2005.

In order to analyze and determine the long term trend, we used the wavelet multiresolution technique (Kumar \& Foufoula-Georgiou 1997; Torrence \& Compo 1998; Percival \& Walden 2000; Souza Echer et al. 2009). The temperature and sunspot number time series were decomposed in orthonormal frequency levels using the discrete Meyer wavelet transform. The approximation level 5 (period $>64$ years) is used in this paper for the study of long term trend in solar activity and Earth's climate.

\section{Results}

Figure 1 shows the long term trend levels for $\mathrm{R}_{z}$, Global (A), $24^{\circ} \mathrm{N}-24^{\circ} \mathrm{S}(\mathrm{B})$, Northern hemisphere (C) and Southern hemisphere (D) averaged surface temperatures.

The Global air surface temperature shows two local maxima, the first one in 1935 and the second one in 2005 (at the end of the series). It also shows two local minima, the first one in 1888, and the second one in 1952 (see Figure 1A). 

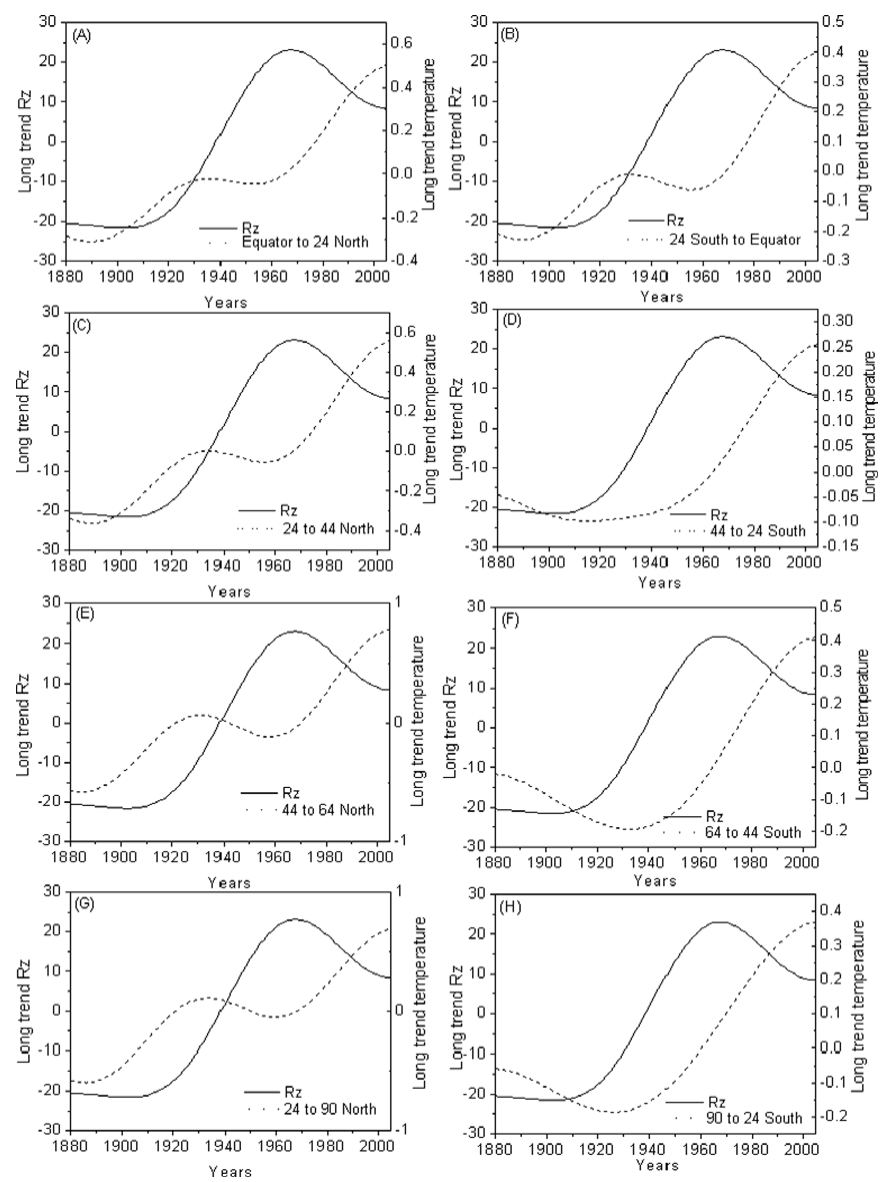

Figure 2. Long term trend for sunspot number (solid line) and for surface temperature (dotted lines). Panels show, on the left, latitudinal trends temperature long term trend for (A) $00^{\circ}-24^{\circ} \mathrm{N}$, (C) $24-44^{\circ} \mathrm{N},(\mathrm{E}) 44-64^{\circ} \mathrm{N}$ and $(\mathrm{G}) 24-90^{\circ} \mathrm{N}$ averaged temperatures; on the right, temperature long term trend for (B) $00^{\circ}-24^{\circ} \mathrm{S}$, (D) $24^{\circ}-44^{\circ} \mathrm{S},(\mathrm{F}) 44-64^{\circ} \mathrm{S}$ and $(\mathrm{H}) 24-90^{\circ} \mathrm{S}$ averaged temperatures

The latitudinal range of $24^{\circ} \mathrm{S}$ to $24^{\circ} \mathrm{N}$ (Figure 1B) shows the same behaviour seen in the global temperature. There are two maxima, the first one in 1934 and the second one in 2005, and two minima, in 1889 and in 1955.

The Northern hemisphere air surface temperature shows a similar long term rising trend. This temperature series has two local maxima, the first one in 1934 and the second one in 2005 (see Figure 1C)

The Southern hemisphere air surface temperature shows that the long term trend is monotonic and upwards from 1880 to 2005. The minimum occurs in 1902 and the maximum in 2005 (see Figure 1D).

The $\mathrm{R}_{z}$ long term curve shows the rising trend from the 1880 until 1970. The plot displays one maximum value in 1968 and two minima. The first minimum occurs in 1903 and the second one in 2005. This long term trend may correspond to the Gleissberg solar cycle (period between 80-100 years).

Figure 2 shows the long term trends for several averaged latitudinal ranges (dotted lines) and again the sunspot number (solid line). See figure caption for identify each latitudinal range. 
Following Figures 1 and 2 we can see that, for both hemispheres there is a rising trend in temperature since 1880. But the shape-behavior is different: for the Northern hemisphere regions there are two maxima in all latitudes. For the Southern hemisphere latitudinal ranges, it seems that most regions have a monotonic curve, with exception of the equatorial region.

\section{Conclusions}

For both hemispheres, there is a rising trend in temperature, since 1880. However, the shape of the long term trend is different. For Northern hemisphere regions, there are two local maxima for all latitudinal ranges. For the Southern hemisphere regions, most latitudinal ranges present a monotonic trend.

The Southern hemisphere temperature series shows a minimum around 1902 and a maximum in 2005. The Northern hemisphere temperature series present two local maxima, in 1934 and in 2005. The temperature series also shows two local minima, the first one in 1886 and the second one in 1958.

The results of this study showed that solar activity and surface temperature have different long term trend over the last 120 years. This means that solar activity effects are not strongly modulating the long term ( $>64$ years) temperature variations, although they can have some effect on the scales of solar 11 and 22 year cycles (e.g, Souza Echer et al. 2009). There are noticeable differences between Northern and Southern hemisphere long term trends, which should be investigated in more detail in future works. Potential factors are regional differences, such as the ocean-land contrast, and anthropogenic effects, as the higher greenhouse gases emissions in Northern hemisphere regions.

\section{Acknowledgements}

The authors would like to thanks CNPq (MPSE: project 156109/2009-8 and 170.180/ 2011-5; EE: project 300211/2008-2; NRR: project 470455/2010), FAPERGS (NRR: project 1013273) and FAPESP (WDG: project 2008/06650-9) agencies for financial support.

\section{References}

Echer, E., Gonzalez, W. D., Guarnieri, F. L., Dal Lago, A., \& Vieira, L. E. A. 2005, Adv. Space Sci.,35, 855

Eddy, J. A. 1976, Science, 192, 1189

Haigh, J. D. 2007, Liv. Rev. Sol. Phys., 4

Hansen, J. \& Lebedeff, S. 1988, Geophys. Res. Lett., 15, 323

Hansen, J., Ruedy, R., Sato, M., \& Reynolds, R. 1996, Geophys. Res. Lett., 23, 1665

Hansen, J., Ruedy, R., Glascoe, J., \& Sato, M. 1999, J. Geophys. Res.,104, 30997

Hathaway, D. H. 2010, Liv. Rev. Solar Phys., 7

Hoyt, D. V. \& Schatten, K. H. 1997, The Role of the Sun in climate change (Oxford University Press)

Kumar, P. \& Foufoula-Georgiou, E. 1997, Rev. Geophys., 35, 385

Percival, D. B. \& Walden, A. T. 2000, Wavelet methods for time series analysis (Cambridge University Press)

Scafetta, N. 2010, J. Atmos. Solar-Terr. Phys., 72, 951

Souza-Echer, M. P., Echer, E., Nordemann, D. J. R., Rigozo, N. R., \& Prestes, A. 2008, Climatic Change, 87, 489 
Souza-Echer, M. P., Nordemann, D. J. R., Echer, E., \& Rigozo, N. R. 2009, J. Atmos. Solar-Terr. Phys., 71, 41

Souza-Echer, M. P., Echer, E., Rigozo, N. R., Brum, C. G. M., Nordemann, D. J. R., \& Gonzalez, W. D 2012, J. Atmos. Solar-Terr. Phys., 74, 87

Torrence, C. \& Compo, G. P. 1998, Bull. American. Meteorol. Soc., 79, 61 